\title{
Meta-learning-based System for solving Logistic Optimization Problems
}

\author{
Alan Davila de León, Eduardo Lalla-Ruiz, Belén Melián-Batista, and J. \\ Marcos Moreno-Vega \\ Department of Computer Engineering and Systems \\ University of La Laguna. La Laguna, Spain \\ \{alan.davila,mbmelian, jmmoreno\}@ull.es \\ Institute of Information Systems \\ University of Hamburg. Hamburg, Germany \\ eduardo.lalla-ruiz@uni-hamburg.de
}

\section{Introduction}

The Algorithm Selection Problem (ASP) is introduced in [5] seeking to answer the question "Which algorithm is the best option to solve my problem?" under those cases where the decision-maker or solver counts with more than one algorithm for a given problem. Formally, the ASP can be defined as follows: having a problem instance $x \in P$, with given features $f(x) \in F$, the objective is to perform a selection mapping $S(f(x))$ in the algorithms space $A$, with the goal of selecting the algorithm $\alpha \in A$ that maximizes the performance mapping $y(\alpha, x) \in Y$ such that $y(\alpha, x) \geq y(a, x), \forall a \in A$. Thus, the principal components of the algorithm selection model are: the problem space $P$, the feature space $F$, the selection mapping $S$ of $P$ on the algorithm space $A$ and the performance space $Y$. The importance of tackling this problem is provided by: $(i)$ No Free Lunch (NFL) theorem, (ii) the big number of available algorithms, and (iii) the need of trying to obtain the best possible solution, and not only a correct one.

In the related literature, some systems have been proposed. The Machine Learning Toolbox (MLT) project [2], continued in Statlog [3] and METAL [1], aims to select the best algorithm for a given dataset. Furthermore, in [1] a helping system for aiding the selection of machine learning algorithms, according to the dataset is proposed. They obtain meta-features that allow to compare different datasets and, by means of that, obtain a reduced group of datasets similar to the one at hand. Those reduced groups are later used to give a recommendation. In [7] a multilayer perceptron network (MLP) is used to select the best optimization algorithm to solve the quadratic assignment problem. However, the above mentioned contributions and systems are focused on recommending or choosing algorithms for a particular problem. That is why a meta-learning ([4]) based system may be appropriate and necessary for those scenarios where a ranking of algorithms sorted according to a provided criterion for any supported input problem is necessary. On the other hand, a drawback appearing in algorithm selection systems is the so-called cold start (see [6]). It concerns the disadvantage that arises in those cases where the system involved in the selection of the 
algorithm for providing a solution has not enough information to give an appropriate recommendation or selection. An extreme case of this problem happens when the system has not past information for comparing the input stream.

\section{Contribution}

Bearing the previous discussion in mind, the main goal of this work is two-fold. On the one hand, a novel meta-learning-based approach that allows to select, from a pool of algorithms, a suitable algorithm for solving a given logistic problem (e.g., vehicle routing problem, berth allocation problem, facility location, etc.) is proposed. On the other hand, the proposed approach is enabled to work within cold start situations where although the system do not have previous information of an introduced logistic problem, it may count with information from a similar problem or from a generalization of it. In doing so, a tree structured hierarchy that allows to compare different metric dataset to identify a particular problem or variation is presented.

\section{References}

1. Brazdil, P.B., Soares, C., Da Costa, J.P.: Ranking learning algorithms: Using ibl and meta-learning on accuracy and time results. Machine Learning 50(3), 251-277 (2003)

2. Kodratoff, Y., Sleeman, D., Uszynski, M., Causse, K., Craw, S.: Building a machine learning toolbox. Enhancing the Knowledge Engineering Process pp. 81-108 (1992)

3. Michie, D., Spiegelhalter, D.J., Taylor, C.C.: Machine learning, neural and statistical classification (1994)

4. Pappa, G.L., Ochoa, G., Hyde, M.R., Freitas, A.A., Woodward, J., Swan, J.: Contrasting meta-learning and hyper-heuristic research: the role of evolutionary algorithms. Genetic Programming and Evolvable Machines 15(1), 3-35 (2014)

5. Rice, J.R.: The algorithm selection problem. Advances in computers 15, 65-118 (1976)

6. Schein, A.I., Popescul, A., Ungar, L.H., Pennock, D.M.: Methods and metrics for cold-start recommendations. In: Proceedings of the 25th annual international ACM SIGIR conference on Research and development in information retrieval. pp. 253260. ACM (2002)

7. Smith-Miles, K.: Towards insightful algorithm selection for optimisation using metalearning concepts. In: WCCI 2008: IEEE World Congress on Computational Intelligence. pp. 4118-4124. IEEE (2008) 